\title{
Supporting information: Machine learning applied to determine the molecular descriptors responsible for the viscosity behavior of concentrated therapeutic antibodies
}

Pin-Kuang Lai ${ }^{\mathrm{a}}$, Amendra Fernando ${ }^{\mathrm{a}}$, Theresa K. Cloutier ${ }^{\mathrm{a}}$, Yatin Gokarn ${ }^{\mathrm{b}}$, Jifeng Zhang ${ }^{\mathrm{b}}$, Walter Schwenger $^{\mathrm{b}}$, Ravi Chari ${ }^{\mathrm{b}, \mathrm{c}}$, Cesar Calero-Rubio ${ }^{\mathrm{b}}$ and Bernhardt L. Trout ${ }^{\mathrm{a}^{*}}$

aDepartment of Chemical Engineering, Massachusetts Institute of Technology, Cambridge, Massachusetts 02139, USA; ${ }^{b}$ Biologics Development, Sanofi, Framingham, MA, USA; 'Current affiliation: Formulation Development, Regeneron

Pharmaceuticals, 777 Old Saw Mill River Rd., Tarrytown, NY 10591, United States (all work performed at Sanofi) 


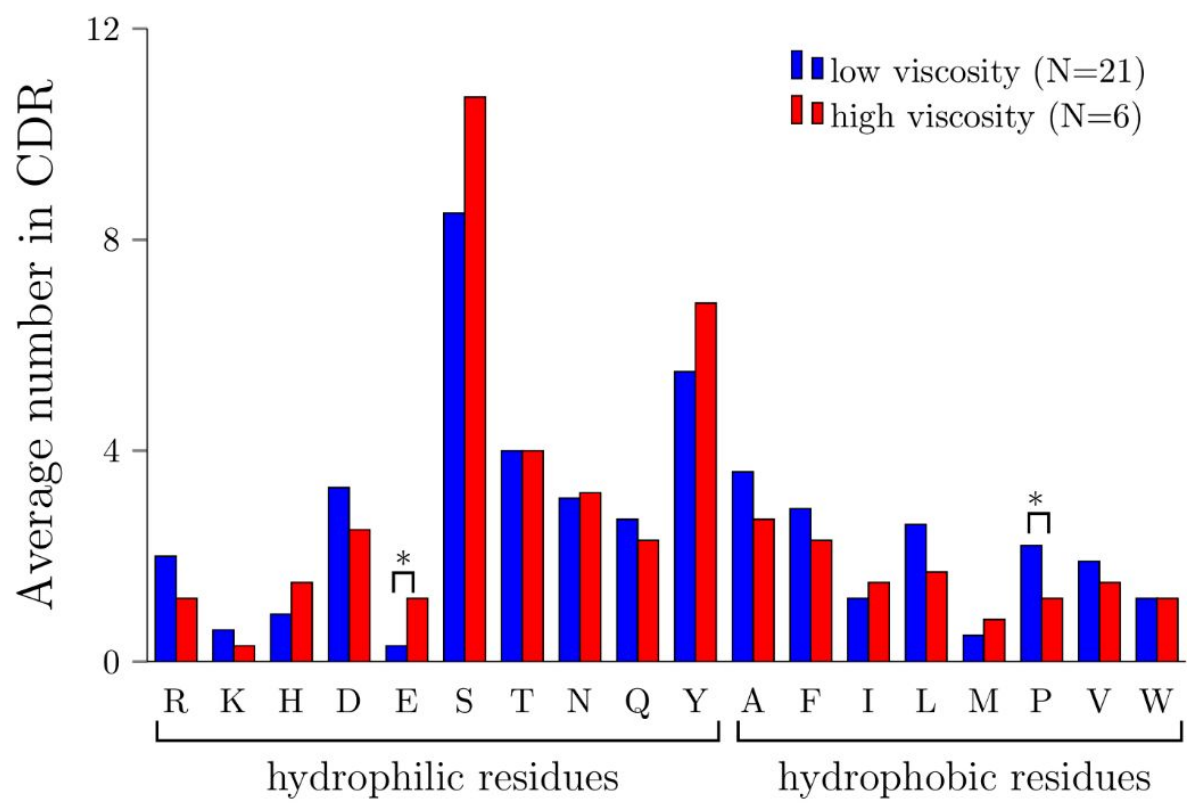

Figure S1. Average number of hydrophilic and hydrophobic residues in the CDR region for the low and high viscosity mAbs. The asterisk symbol indicates $\mathrm{p}$-value $<0.05$. 


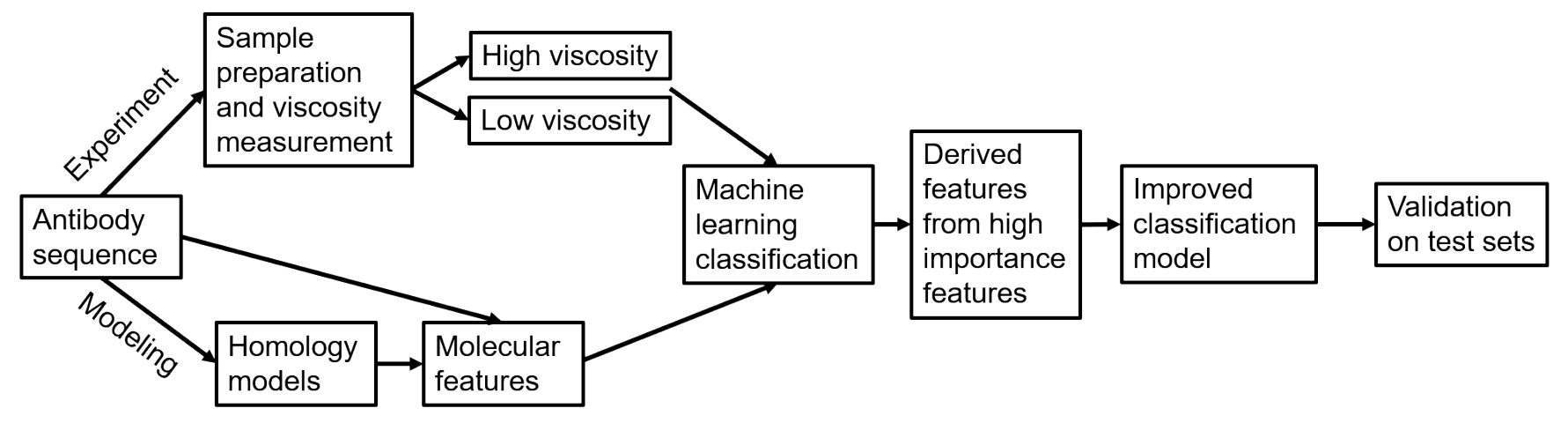

Figure S2. Frame diagram of the experimental design and machine learning approach. 
Table S1. The 27 FDA approved mAbs used in this study and their molecular properties and prediction scores.

\begin{tabular}{|c|c|c|c|c|c|}
\hline $\mathrm{mAb}$ & Isotype & $\begin{array}{c}\text { net } \\
\text { charge }\end{array}$ & SCM & HVI & $\begin{array}{c}\text { viscosity } \\
150 \mathrm{mg} / \mathrm{mL}\end{array}$ \\
\hline mAb1 & $\operatorname{IgG} 1 \kappa$ & 26 & 1261.4 & 16.7 & 14.4 \\
\hline mAb2 & $\operatorname{IgG} 1 \kappa$ & 22 & 843.9 & 12.4 & 20.9 \\
\hline mAb3 & $\operatorname{IgG} 1 \kappa$ & 26 & 1085.0 & 19.1 & 14.9 \\
\hline $\mathrm{mAb} 4$ & $\operatorname{IgG} 1 \kappa$ & 20 & 1203.1 & 20.4 & 93.4 \\
\hline mAb5 & $\operatorname{IgG} 1 \kappa$ & 26 & 818.5 & 10.0 & 8.6 \\
\hline mAb6 & $\operatorname{IgG} 2 \kappa$ & 24 & 959.9 & 13.9 & 9.0 \\
\hline $\mathrm{mAb7}$ & $\operatorname{IgG} 2 / 4 \kappa$ & 4 & 1293.9 & 16.2 & 29.0 \\
\hline mAb8 & $\operatorname{IgG} 1 \kappa$ & 22 & 1049.2 & 14.6 & 12.9 \\
\hline mAb9 & $\operatorname{IgG} 2 \lambda$ & 20 & 1074.0 & 22.8 & 52.3 \\
\hline mAb10 & $\operatorname{IgG} 1 \kappa$ & 34 & 736.2 & 13.2 & 10.2 \\
\hline $\mathrm{mAb} 11$ & $\operatorname{IgG} 1 \kappa$ & 16 & 1038.4 & 23.8 & 100.3 \\
\hline mAb12 & $\operatorname{IgG} 1 \kappa$ & 30 & 873.4 & 14.7 & 7.5 \\
\hline mAb13 & $\operatorname{IgG} 4 \kappa$ & 14 & 938.3 & 16.4 & 12.5 \\
\hline $\mathrm{mAb} 14$ & $\operatorname{IgG} 1 \kappa$ & 24 & 1067.0 & 13.4 & 23.4 \\
\hline $\mathrm{mAb} 15$ & $\operatorname{IgG} 1 \kappa$ & 32 & 736.6 & 16.7 & 12.9 \\
\hline mAb16 & $\operatorname{IgG} 1 \kappa$ & 26 & 1053.7 & 15.3 & 10.0 \\
\hline $\mathrm{mAb} 17$ & $\operatorname{IgG} 1 \kappa$ & 20 & 1316.2 & 21.6 & 210.9 \\
\hline mAb18 & $\operatorname{IgG} 1 \kappa$ & 28 & 789.3 & 15.0 & 7.1 \\
\hline mAb19 & $\operatorname{IgG} 2 \kappa$ & 10 & 1205.0 & 19.0 & 21.2 \\
\hline $\mathrm{mAb} 20$ & $\operatorname{IgG} 4 \kappa$ & 12 & 1158.4 & 19.0 & 24.4 \\
\hline $\mathrm{mAb} 21$ & $\operatorname{IgG} 1 \kappa$ & 24 & 981.3 & 17.3 & 8.6 \\
\hline $\mathrm{mAb} 22$ & $\operatorname{IgG} 1 \kappa$ & 30 & 950.6 & 13.0 & 9.0 \\
\hline mAb23 & $\operatorname{IgG} 1 \kappa$ & 30 & 741.7 & 17.2 & 22.9 \\
\hline $\mathrm{mAb} 24$ & $\operatorname{IgG} 1 \kappa$ & 26 & 1002.9 & 17.3 & 90.5 \\
\hline $\mathrm{mAb} 25$ & $\operatorname{IgG} 1 \kappa$ & 34 & 853.5 & 22.1 & 7.4 \\
\hline $\mathrm{mAb} 26$ & $\operatorname{IgG} 1 \kappa$ & 26 & 935.1 & 17.2 & 10.3 \\
\hline $\mathrm{mAb} 27$ & $\operatorname{IgG} 1 \kappa$ & 26 & 1212.0 & 19.3 & 103.8 \\
\hline
\end{tabular}


Table S2. Concentration dependent viscosity measurement on the $27 \mathrm{mAbs}$ used in this study. Each viscosity value is an average of 2 independent measurements. The unit for concentration is in $\mathbf{m g} / \mathbf{m L}$, and the unit for viscosity is in $\mathbf{c P}$.

\begin{tabular}{ccccccccccccc}
\hline mAbs & C50 & $\eta$ & C100 & $\eta$ & C125 & $\eta$ & C150 & $\eta$ & C175 & $\eta$ & C200 & $\eta$ \\
\hline mAb1 & 55 & 1.8 & 108 & 4.4 & 133 & 8 & 160 & 15.0 & 180 & 32.5 & 206 & 70.5 \\
mAb2 & 52 & 2.0 & 104 & 6.1 & 131 & 12 & 153 & 22.1 & 176 & 44.5 & 207 & 86.5 \\
mAb3 & 56 & 1.8 & 108 & 4.5 & 136 & 9 & 158 & 17.1 & 177 & 30.0 & 209 & 75.0 \\
mAb4 & 52 & 3.9 & 108 & 25.0 & 133 & 59 & 156 & 119.5 & 182 & 267.0 & 209 & 541.0 \\
mAb5 & 56 & 1.6 & 108 & 3.4 & 137 & 5 & 144 & 7.6 & 179 & 14.8 & 198 & 28.7 \\
mAb6 & 54 & 1.7 & 108 & 3.0 & 129 & 5 & 151 & 8.3 & 177 & 13.9 & 203 & 37.0 \\
mAb7 & 56 & 2.0 & 103 & 4.8 & 131 & 19 & 152 & 22.9 & 182 & 76.0 & 204 & 200.0 \\
mAb8 & 54 & 1.8 & 110 & 4.3 & 134 & 8 & 154 & 12.8 & 183 & 24.9 & 194 & 47.7 \\
mAb9 & 53 & 1.9 & 112 & 7.7 & 127 & 20 & 160 & 76.0 & 182 & 226.0 & 204 & 400.0 \\
mAb10 & 52 & 1.7 & 105 & 3.3 & 133 & 6 & 156 & 10.3 & 178 & 16.8 & 202 & 41.0 \\
mAb11 & 53 & 2.4 & 103 & 13.9 & 126 & 36 & 144 & 117.0 & 169 & 237.0 & 192 & 395.0 \\
mAb12 & 55 & 1.7 & 95 & 2.7 & 122 & 4 & 150 & 6.6 & 167 & 9.7 & 203 & 23.4 \\
mAb13 & 59 & 1.8 & 101 & 3.5 & 129 & 7 & 151 & 12.0 & 182 & 28.7 & 201 & 42.0 \\
mAb14 & 53 & 1.9 & 107 & 6.1 & 131 & 12 & 152 & 24.4 & 185 & 61.5 & 206 & 126.0 \\
mAb15 & 53 & 2.0 & 105 & 3.9 & 105 & 4 & 153 & 10.6 & 176 & 22.4 & 211 & 70.0 \\
mAb16 & 58 & 1.8 & 111 & 3.9 & 124 & 5 & 157 & 10.6 & 170 & 15.0 & 206 & 38.0 \\
mAb17 & 46 & 3.5 & 98 & 37.0 & 123 & 120 & 147 & 265.0 & 175 & 488.0 & 214 & 1400.0 \\
mAb18 & 55 & 1.8 & 108 & 3.3 & 134 & 5 & 158 & 7.0 & 179 & 11.8 & 206 & 20.5 \\
mAb19 & 56 & 1.8 & 109 & 5.5 & 138 & 12 & 155 & 22.7 & 170 & 45.0 & 200 & 98.0 \\
mAb20 & 57 & 12.0 & 94 & 7.4 & 119 & 12 & 151 & 18.1 & 176 & 35.2 & 207 & 82.0 \\
mAb21 & 53 & 1.7 & 108 & 3.4 & 129 & 5 & 151 & 7.7 & 177 & 14.4 & 203 & 28.0 \\
mAb22 & 57 & 1.8 & 108 & 3.7 & 129 & 5 & 155 & 8.8 & 184 & 15.6 & 203 & 33.5 \\
mAb23 & 54 & 1.9 & 105 & 4.9 & 130 & 10 & 147 & 19.7 & 176 & 52.5 & 201 & 121.0 \\
mAb24 & 58 & 3.3 & 100 & 14.8 & 121 & 40 & 152 & 94.0 & $\mathrm{~N} / \mathrm{A}$ & $\mathrm{N} / \mathrm{A}$ & 207 & 640.0 \\
$\mathrm{mAb} 25$ & 51 & 1.6 & 102 & 3.0 & 129 & 4 & 148 & 6.8 & 171 & 10.7 & 194 & 19.5 \\
$\mathrm{mAb26}$ & 52 & 2.0 & 103 & 3.9 & 129 & 6 & 154 & 10.0 & 173 & 16.0 & 198 & 30.0 \\
$\mathrm{mAb27}$ & $\mathrm{N} / \mathrm{A}$ & $\mathrm{N} / \mathrm{A}$ & $\mathrm{N} / \mathrm{A}$ & $\mathrm{N} / \mathrm{A}$ & 112 & 35 & 147 & 85.0 & 171 & 210.0 & 199 & 440.0 \\
\hline
\end{tabular}


Table S3. The five metrics of Therapeutic Antibody Profiler (TAP) and viscosity for the $27 \mathrm{mAbs}$ in this study. The colors for the five metrics follow the definition from the TAP server. Low and high viscosity mAbs are assigned with green and red flags, respectively.

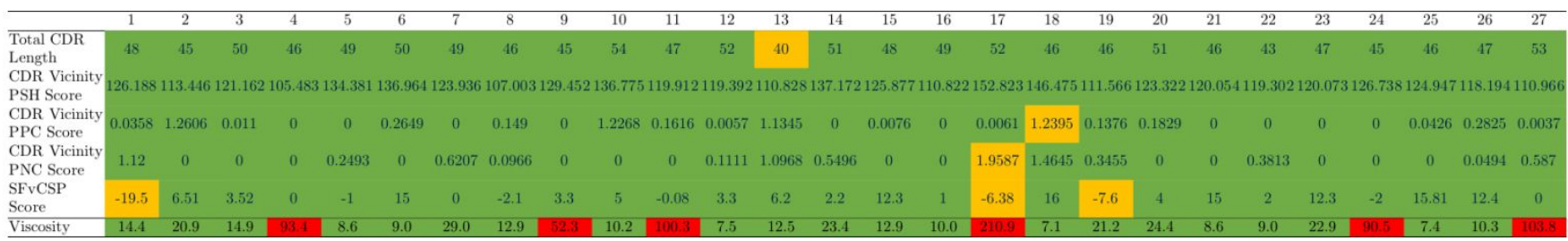

